
Cahiers
de a Recherche
surles Droits
Fondamentaux

\section{Cahiers de la recherche sur les droits} fondamentaux

13 | 2015

Le droit d'asile

\title{
De l'instruction des demandes à l'établissement de
}

faits

From the Consideration of Requests to the Establishment of Facts

Jean Kohler

\section{CpenEdition}

1 Journals

Édition électronique

URL : http://journals.openedition.org/crdf/1158

DOI : $10.4000 /$ crdf. 1158

ISSN : 2264-1246

Éditeur

Presses universitaires de Caen

Édition imprimée

Date de publication : 1 novembre 2015

Pagination : $31-37$

ISBN : 978-2-84133-742-2

ISSN : $1634-8842$

\section{Référence électronique}

Jean Kohler, "De l'instruction des demandes à l'établissement de faits », Cahiers de la recherche sur les droits fondamentaux [En ligne], 13 | 2015, mis en ligne le 01 novembre 2016, consulté le 11 février 2020. URL : http://journals.openedition.org/crdf/1158; DOI : 10.4000/crdf.1158 


\title{
De l'instruction des demandes à l'établissement de faits
}

\author{
Jean KOHLER \\ Juriste, chef de service du centre d'accueil de demandeurs d'asile (CADA) de Saint-Denis
}

\begin{abstract}
I. Les problématiques structurelles de la demande d'asile
II. La définition préalable du réfugié, une personne étrangère

III. Une procédure d'instruction à délai limité

IV. Le non-établissement des faits étrangers

V. L'oubli de l'acte de persécution dans la demande d'asile

VI. L'application du droit pénal en compétence universelle pour certains cas graves

VII. Une procédure d'asile sous forme de procédure pénale ad hoc?
\end{abstract}

\section{Les problématiques structurelles de la demande d'asile}

À la lumière des images médiatiques de ces derniers mois, comptabilisant le nombre de morts naufragés sur nos côtes européennes, il pourrait être facile de penser que nous sommes à l'aube d'un nouveau monde où les personnes étrangères seraient de plus en plus nombreuses à immigrer sur notre territoire. Lors du dépôt du projet de loi sur l'asile ${ }^{1}$, les chiffres cités parlaient d'eux-mêmes: en 2007 environ 35000 demandes d'asile alors qu'en 2013 la France totalisait près de 65000 demandes d'asile. La peur et l'angoisse pouvaient ainsi envahir l'opinion publique, propices à l'adoption d'un projet de loi que l'ensemble des professionnels du secteur dénonce. Néanmoins, avec un œil aguerri sur la situation de l'accueil des demandeurs d'asile en France, nous pouvons observer très facilement que nous avons récupéré un nombre de demandeurs d'asile égal à celui des années 2003 et 2004. Cependant, le traitement des demandes d'asile a, comme l'ensemble des délégués de service public, subi une baisse significative de ses moyens financiers. Pour les centres d'accueil de demandeurs d'asile (CADA), cette baisse de moyens s'est traduite par l'augmentation du nombre de résidents pris en charge pour un emploi à temps plein (ETP). Nous sommes actuellement à un ETP pour dix à quinze alors qu'avant 2011 nous étions à un ETP pour dix. Il faut savoir que chaque CADA dispose d'un budget propre alloué et contrôlé par la direction régionale et interdépartementale de l'hébergement et du logement (DRIHL). Ce nombre de résidents pour un ETP se traduit par une diminution de l'allocation générale accordée à un demandeur d'asile dans sa prise en charge globale d'accueil. L'accompagnement personnalisé de ces demandeurs est dès lors de plus en plus difficile.

1. Projet de loi relatif à la réforme de l'asile, $\mathrm{n}^{\circ} 2182$, enregistré à la présidence de l'Assemblée nationale le 23 juillet 2014 , en ligne: http://www. assemblee-nationale.fr/14/projets/pl2182.asp. Pour un suivi de l'état du projet de loi et ses amendements en commission, voir http://www.gisti. org/spip.php?articlezozo. 
Ayant travaillé trois années en tant que juriste au sein d'un centre d'accueil pour demandeur d'asile, j'ai été aux

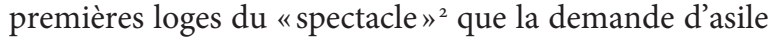
donnait aux personnes fuyant des crimes et demandant une protection au pays des droits de l'homme. Malheureusement, j'ai fait l'amer constat que des demandeurs d'asile, ayant vécu des horreurs dans leur pays d'origine, ne se sont pas vu reconnaître le statut de réfugié tandis que d'autres, dont le récit de vie était plus que douteux, se voyaient accorder une protection. Ce constat a été fait de nombreuses fois au cours de mes années en qualité de juriste, et ces décisions ont toutes fait preuve d'un manque manifeste de motivation dans la rédaction des motifs de rejet. Le pire dans ces situations ubuesques a été le fait que l'ensemble de ces personnes déboutées de leur demande d'asile ont obtenu, par la suite, une carte de séjour pour soins $^{3}$ en raison des troubles post-traumatiques graves qu'ils avaient subis. Les médecins de l'agence régionale de santé (ARS), responsables de l'examen des demandes de carte de séjour pour soins, avaient analysé le dossier médical plus justement que les juges de la Cour nationale du droit d'asile (CNDA) lors de l'audience. En effet, l'analyse des situations médicales, opérée par l'Office français de protection des réfugiés et apatrides (OFPRA) ou la CNDA, est une problématique essentielle dans la qualité de la demande d'asile. Nous savons qu'il n'y a presque pas d'expertise médicale ordonnée par l'OFPRA ou la CNDA afin de confirmer les dires des certificats médicaux à l'appui des demandes d'asile, pourtant ces mêmes certificats sont écartés par les officiers et les juges dans leurs décisions.

Sur le fond des décisions, les autorités françaises sont reconnues comme ne remplissant pas les conditions minimums de respect des droits de la défense et d'une procédure juste et équitable. Les différentes condamnations de la Cour européenne des droits de l'homme (Cour EDH) ${ }^{4}$, ou des différents organismes de défense des droits de l'homme, soulignent les mauvaises applications du droit d'asile au travers, d'une part, de décisions sans réelle motivation ou, d'autre part, de procédures contraires à certains droits fondamentaux.

Les différentes procédures appliquées aux demandeurs d'asile ne favorisent pas non plus une instruction juste et équitable. La procédure de la demande d'asile, la condition de la forme d'accès au droit d'asile, a été à l'origine du dispositif européen de l'asile. Les premières dispo- sitions communautaires en matière d'asile ont concerné la convention de Dublin avec sa transposition actuelle en règlement Dublin III ${ }^{5}$. Cette première règle européenne dispose que le demandeur d'asile ne peut introduire une demande d'asile que dans le premier pays dans lequel il est arrivé dans l'Union européenne (UE). Les pays de l'UE avec des frontières extérieures se retrouvent en première ligne et doivent assumer l'ensemble des demandes d'asile. La Bulgarie, pour exemple, est la porte d'entrée de l'UE sur le chemin d'exil des Syriens fuyant la guerre. Son système d'accueil de l'asile n'étant pas adapté pour un tel afflux, les autorités ont dû cesser de répondre positivement aux demandes de renvoi des Syriens "dublinés ${ }^{6}$. En janvier 2014, le Haut-Commissariat pour les réfugiés de l'Organisation des Nations unies (HCR) a même dû exhorter les pays européens à suspendre le renvoi sous la procédure Dublin de demandeurs d'asile en raison d'un risque certain de mauvais traitement ${ }^{7}$.

La procédure dite "prioritaire ${ }^{8}$ est un autre exemple des conséquences d'une procédure sur le fond de l'application du droit d'asile. Lorsqu'un demandeur d'asile vient d'un pays qui est considéré comme sûr, ou peut représenter une menace grave pour l'ordre public, ou encore présente une demande d'asile manifestement abusive, il sera alors placé en procédure prioritaire. L'OFPRA devra prendre une décision dans les quinze jours ou dans les quatre-vingtseize heures s'il est en rétention. Le temps nécessaire pour faire des recherches sur la réalité des faits est très faible en cas de demande manifestement non fraudée. La CNDA, qui procède à l'examen des recours contre les décisions de rejet de l'OFPRA, ne donne pas de meilleures garanties des droits aux demandeurs d'asile. Il faut noter qu'en cas de procédure prioritaire, le recours devant la CNDA n'est pas suspensif d'une mesure d'éloignement. Certes, au regard du projet de loi actuel, il y aurait un recours suspensif devant la CNDA prévu pour la procédure dite «accélérée» qui remplacera la procédure prioritaire mais, en contrepartie, la CNDA pourra siéger à juge unique. L'absence de collégialité n'est pas la meilleure garantie pour une défense effective des droits fondamentaux. La procédure accélérée prévoirait un nombre croissant de cas lui permettant d'être appliquée ${ }^{9}$. Alors que la procédure prioritaire avait démontré ses lacunes, le projet de loi a décidé de renforcer ce dispositif décrié par tous comme une procédure à garanties diminuées.

2. Terme utilisé au sujet de la Cour nationale du droit d'asile (CNDA) par une majorité des demandeurs d'asile déboutés de leur demande.

3. Au sens de l'article $313-1111^{\circ}$ du Code de l'entrée et du séjour des étrangers et du droit d'asile (CESEDA).

4. Cour EDH, 15 janvier 2015, A. A. c. France, $\mathrm{n}^{\circ} 18039 / 11$.

5. Le règlement UE no 604/2013 du Parlement et du Conseil du 26 juin 2013, dit règlement Dublin III, fixe les critères et mécanismes objectifs et équitables permettant de déterminer l'État membre responsable de l'examen d'une demande d'asile présentée dans l'un des États membres par un ressortissant d'un État tiers.

6. Terme faisant référence à l'application de la procédure Dublin pour signifier qu'un demandeur d'asile, qui s'est rendu dans un deuxième pays européen, sera renvoyé dans le premier pays européen. Les autorités du pays responsable de la demande peuvent refuser ou simplement ne pas donner suite à la demande de renvoi du «Dubliné» formulée par les autorités du deuxième pays.

7. Voir le rapport du HCR, Observations sur la situation actuelle de l'asile en Bulgarie, 2 janvier 2014, et l'article «Le HCR et l'ONG ECRE appellent à la suspension temporaire des transferts "Dublin" vers la Bulgarie», en ligne: http://www.forumrefugies.org/s-informer/actualites/ le-hcr-et-l-ong-ecre-appellent-a-la-suspension-temporaire-des-transferts-dublin-vers-la-bulgarie.

8. Art. L. 741-4 CESEDA.

9. Là où la procédure prioritaire s'appliquait à quatre cas, la procédure accélérée en prévoirait neuf. 


\section{La définition préalable du réfugié, une personne étrangère}

Alors que l'application du droit d'asile, et la procédure qui l'entoure, ne permettent pas de garantir des droits suffisants pour une reconnaissance juste et équitable du statut de réfugié, il est légitime de s'interroger sur les raisons de ces lacunes dans la défense de nos droits fondamentaux. En comparant la situation d'un demandeur d'asile avec celle d'un ressortissant français ou européen, nous pouvons constater que le traitement n'est pas le même. En effet, une femme qui se dit victime d'un viol, ou un homme qui allègue avoir été torturé, n'aura pas le droit à la même prise en charge s'il est français ou étranger. Cette logique découle du principe de protection de ses ressortissants, naturellement inscrit dans le droit à la nationalité et dans les fondements de l'État souverain. En matière d'asile, le défaut de protection de l'État d'origine est un des critères principaux pour la reconnaissance $\mathrm{du}$ statut de réfugié. En sus, si une personne craint d'être persécutée en raison de l'un des cinq motifs énumérés par la Convention de Genève de $1951^{10}$, elle sera reconnue comme réfugiée et prise en charge par l'état d'accueil. Dans cette optique l'état d'accueil va se substituer à l'état d'origine du réfugié en lui fournissant les services d'état civil et en lui assurant les mêmes droits que ceux accordés aux nationaux. Ainsi les réfugiés reconnus ont droit à la même protection de la part de l'État que celle accordée aux nationaux. Dans l'attente de leur reconnaissance, la situation est beaucoup plus contrastée pour les demandeurs d'asile. Dans cette situation, il ne faut pas avoir peur des mots en affirmant que la différence d'approche réside dans le fait qu'elle soit étrangère ou non. Le demandeur d'asile reste avant tout un étranger. L'article premier de la Convention de Genève dispose que «le terme "réfugié" s'appliquera à toute personne» qui remplit les conditions énumérées après. Le vecteur de qualification du droit d'asile réside en la personne. La directive dite "Qualification» de 2004 confirme cette approche en posant la définition dans son article premier des demandeurs d'asile en tant que ressortissants de pays tiers, apatrides ou simplement personnes qui ont besoin d'une protection ${ }^{11}$. L'approche du demandeur d'asile en tant que personne, et in extenso en tant qu'étranger, est finalement une vision essentielle et permanente dans la procédure d'asile.

\section{Une procédure d'instruction à délai limité}

La procédure d'asile souffre d'un délai de traitement des demandes trop long. Le débat actuel sur le projet de loi sur l'asile dispose qu'il y aura une réduction des délais et fixe pour ce faire des durées de traitement à respecter. Certains demandeurs de mon centre sont restés jusqu'à quatre années pris en charge dans l'attente d'une réponse définitive. Avec souvent une année précédant l'arrivée en CADA, il est difficile de ne pas reconnaître une intégration dans la société française après cinq années de présence sur le territoire, permettant le plus souvent d'obtenir une carte de séjour ${ }^{12}$. La procédure d'asile est ainsi un moyen d'obtenir du temps pour marquer son séjour sur le territoire, ce qui fait qu'elle est autant exposée à la fraude. Le coût d'une prise en charge pour une famille en matière d'hébergement, allocations, et suivi social, emporte, avec ce système favorisant la fraude, la motivation politique de raccourcir les délais sur les raisons impérieuses de réduction des dépenses publiques. Pour autant, aux yeux des professionnels du secteur, les raisons des délais si longs, qui pèsent sur les institutions comme sur les usagers, sont à chercher dans les moyens accordés aux institutions responsables de l'analyse de la demande d'asile. L'OFPRA et la CNDA croulent sous les demandes sans avoir les effectifs nécessaires pour les prendre en charge. Les revendications de certains des professionnels de ces institutions ont entraîné une mobilisation visant notamment à demander un renforcement des effectifs. La grève des rapporteurs de la CNDA en février 2015 a illustré ce besoin primaire de tous les professionnels du secteur de l'asile: augmenter le nombre d'agents. Malheureusement, le nombre de rapporteurs auprès de la CNDA ne va pas sensiblement changer si l'on regarde la retranscription de la loi de finances 2015. Alors que l'objectif de réduction des délais est clairement prôné, le nombre de rapporteurs a seulement été augmenté de cinq nouveaux postes pour l'année $2015^{13}$. Cette réalité

10. Le réfugié est défini comme une personne qui « [...], craignant avec raison d’être persécutée du fait de sa race, de sa religion, de sa nationalité, de son appartenance à un certain groupe social ou de ses opinions politiques, se trouve hors du pays dont elle a la nationalité et qui ne peut ou, du fait de cette crainte, ne veut se réclamer de la protection de ce pays; ou qui, si elle n'a pas de nationalité et se trouve hors du pays dans lequel elle avait sa résidence habituelle à la suite de tels événements, ne peut ou, en raison de ladite crainte, ne veut y retourner» (art. 1.A.2 de la Convention de Genève du 28 juillet 1951 relative au statut des réfugiés; ci-après « Convention de Genève»).

11. «La présente directive a pour objet d'établir des normes minimales relatives aux conditions que doivent remplir les ressortissants de pays tiers ou les apatrides pour pouvoir prétendre au statut de réfugié ou les personnes qui, pour d'autres raisons, ont besoin d'une protection internationale, et relatives au contenu de la protection accordée» (directive 2004/83/CE dite "Qualification", 29 avril 2004).

12. Au regard des conditions de la circulaire du ministère de l'Intérieur du 28 novembre $2012, n^{\circ}$ INTK1229185C, cinq années de présence en France sont nécessaires pour demander une régularisation par le travail, ou par l'intégration des enfants dans la société.

13. Projet de loi de finances pour 2015: Asile, enregistré à la présidence du Sénat le 20 novembre 2014, en ligne: http://www.senat.fr/rap/a14-114-2/ a14-114-23.html: «Les crédits de titre 2 qui sont alloués à la CNDA figurent à l'action 7 du programme no 165 : “Conseil d'État et autres juridictions administratives" de la mission "Conseil et contrôle de l'État". Ces crédits connaîtront en 2015 une augmentation de près de 3 \% pour atteindre 22,87 millions d'euros. Cette progression des crédits de rémunération correspond à la création de 9 EPTP. [...] Le triennal $2015-2017$ prévoit à nouveau la création de 27 ETPT, dont 6 de magistrats, 15 de rapporteurs et 6 d'agents de greffe. Les 9 ETPT créés cette année permettront dès 2015 le recrutement de 2 magistrats et 7 agents ». 
amène à se poser la question des moyens employés par rapport aux fins visées. Comme le dénonçait un rapporteur, ces derniers sont soumis à une obligation de quantité et non de qualité, ce qui les oblige actuellement d'instruire environ 370 demandes par an et par rapporteur ${ }^{14}$. Il est donc légitime, aux regards des objectifs du projet de loi, de craindre pour ces professionnels une augmentation de la quantité des dossiers à traiter au détriment de la qualité de leur travail.

Les officiers de protection de l'OFPRA sont soumis eux aussi à une obligation de résultat de deux décisions par jour. Malgré la différence nette de traitement des demandes entre les deux types de procédures (normale et prioritaire), cette obligation s'applique sans distinction sur la base d'un quota annuel. Autant en procédure prioritaire qu'en procédure normale, les décisions doivent être prises sur les dossiers triés et distribués par le chef de section. Ce dernier est responsable du partage des dossiers sur la base de l'expérience, mais aussi du processus d'attribution d'une protection par ses officiers. Il détient un droit de regard obligatoire sur l'ensemble des décisions et peut ainsi refuser de reconnaître le statut, alors que l'officier souhaitait le contraire. Simplement au regard des éléments du dossier et sans avoir jamais entendu le demandeur, le chef de section peut rejeter une demande sur la base de ses connaissances acquises par son expérience professionnelle en tant qu'ancien officier de protection. Certes sa présence est un atout pour le dialogue constructif d'une instruction, et il peut aussi demander un deuxième entretien ou un complément d'instruction dans les cas de clauses d'exclusion par exemple. Cependant, il ne devrait pas a priori pouvoir aussi formellement s'exprimer dans le processus décisionnel si l'on souhaite réellement maintenir l'indépendance de l'instruction des officiers de protection. Cette obligation de résultat soumet l'instruction menée par les officiers à un objectif prioritaire de quantité, sous le contrôle hiérarchique de leurs supérieurs.

La procédure se doit ainsi d'être transparente et respecter le principe du contradictoire pour permettre de lever les doutes des autorités lors d'un entretien ou d'un recours contre une décision de rejet. Malgré le fait que la charge de la preuve pèse sur le demandeur, l'État d'accueil a cependant une obligation d'apporter des éléments étayant ses doutes concernant la véracité de la demande d'asile. En l'absence de ces éléments, le bénéfice du doute doit être accordé au demandeur. La difficulté en France dans l'application de ces principes dans la procédure est que l'État d'accueil est juge et partie à la fois. En effet, lorsque vous introduisez une demande devant l'OFPRA, cette administration représente la
France, pays d'accueil, mais aussi l'autorité responsable de l'analyse de la demande d'asile. L'instruction se fait donc principalement à charge et rarement à décharge, mettant ainsi le demandeur d'asile en déséquilibre face à l'égalité des armes, condition absolue d'un procès équitable. Devant la CNDA, rares sont les audiences où un avocat représentant l'OFPRA est présent. Dans ce système bipartite, les demandeurs sont face à des juges mais aussi à des contradicteurs.

Les garanties procédurales imposent clairement un objectif de gestion de flux migratoires et limitent l'accès à une instruction juste et équitable.

\section{Le non-établissement des faits étrangers}

Sur le fond, les règles encadrant la reconnaissance du statut de réfugié ne sont pas plus valorisantes pour une instruction juste et équitable. Les décisions de rejet de la part de la CNDA et de l'OFPRA se voient régulièrement épinglées par les juridictions européennes pour un manque de motivation trahissant un manque d'instruction de la part des autorités ${ }^{15}$. Rejetant des demandes d'asile sur les motifs que le récit était stéréotypé ou peu détaillé, cette argumentation, propre à l'OFPRA et à la CNDA, a été reprise par la Cour EDH pour qualifier les décisions émises par les autorités françaises.

Pour la juridiction strasbourgeoise, il semblait qu'en l'état des débats politiques actuels et après une première série d'arrêts datés de 2013, la CNDA et l'OFPRA avaient adopté de nouvelles formules pour motiver leurs décisions de rejet. Malheureusement, les motivations simples et stéréotypées des décisions sont toujours présentes. Par conséquent, la Cour européenne des droits de l'homme a de nouveau souligné la violation de la convention par la France, dans un double arrêt en date du 15 janvier $2015^{16}$, pour des motivations de décisions insuffisamment détaillées et personnalisées. La Cour européenne a eu à se prononcer sur la conformité de l'article 3 de la Convention européenne des droits de l'homme (Convention $\mathrm{EDH})^{17}$ avec des mesures d'éloignement prises à l'encontre de Soudanais. Par ricochet, elle a analysé la décision de rejet de la demande d'asile à la base de la mesure d'éloignement. Le constat opéré par la Cour européenne soulève une pratique de la part de l'OFPRA et de la CNDA, qui ne justifient pas suffisamment leurs décisions de rejet. La Cour soulève les formules stéréotypées des décisions des autorités françaises qui ne permettent pas aux demandeurs d'émettre une objection claire et objective lors d'un recours éventuel. Les garanties

14. S. Mouillard, «Droit d'asile: on travaille toujours à flux tendu», Libération, 3 février 2015, en ligne: http://www.liberation.fr/societe/2015/o2/o3/ droit-d-asile-on-travaille-toujours-a-flux-tendu_1194816.

15. Cour EDH, 10 octobre 2013, K. K. c. France, $\mathrm{n}^{\circ}$ 18913/11; Cour EDH, 14 novembre 2013, Z. M. c. France, $\mathrm{n}^{\circ}$ 40042/11; Cour EDH, 19 décembre 2013, N. K. c. France, $\mathrm{n}^{\circ} 7974 / 11$.

16. Cour EDH, 15 janvier 2015, A. A c. France, $\mathrm{n}^{\circ}$ 18039/11; A. F. c. France, $\mathrm{n}^{\circ} 80086 / 13$

17. Art. 3 de la Convention EDH. 
d'authenticité des documents à l'appui sont considérées abusivement comme insuffisantes et ne permettant pas de corroborer les faits allégués. Les justifications pour écarter certains documents à l'appui ont été condamnées par la Cour dans les arrêts de 2013, et de nouveau dans les deux derniers de janvier 2015.

Au sein de notre CADA, nous avons reçu de nombreuses décisions de rejet sur la base de motifs vagues et incompréhensibles. Certaines étaient tout simplement hallucinantes du fait de la méconnaissance de la situation du pays ou du demandeur.

À titre d'exemple, un jeune Guinéen membre d'un parti d'opposition se disait persécuté par l'unité de lutte antidrogue dirigée par $\mathrm{M}$. Tiegboro. Il a reçu sa décision de rejet de l'OFPRA sur le motif que ce M. Tiegboro n'est plus en poste car soumis à une enquête interne par les autorités de son pays depuis 2011. Le jeune Guinéen m’explique, désespéré, que, certes il est bien mis en cause par une commission d'enquête nationale, mais qu'il est encore au pouvoir au sein de son unité de lutte antidrogue. Je fais quelques recherches sur Internet et trouve une multitude d'articles de presse expliquant que ce M. Tiegboro est encore le directeur effectif de l'unité de lutte antidrogue en Guinée. Il m'aura fallu cinq minutes pour contredire l'argument majeur de rejet d'une décision que l'OFPRA a mis six mois à prendre. Cet événement désespérant peut paraître anodin ou épisodique, mais lors de mes trois années en tant que juriste, ces situations ont été trop souvent récurrentes et ont créé à chaque fois un grand désespoir chez les demandeurs d'asile rejetés. Je me souviens d'une audience CNDA où la présidente confondait le nom de l'opposant politique avec le nom du dictateur au pouvoir. Dans ces circonstances, une incompréhension suivie d'une frustration et un sentiment d'impuissance s'installent chez les demandeurs d'asile qui n'ont pas de justification suffisante au rejet de leur demande.

Le non-respect des recommandations européennes en matière de motivation des décisions trahit ce manque d'instruction qu'observent les demandeurs lors de la notification de leur décision de rejet. Lorsqu'on a fui son pays, sa famille et sa culture, en raison de persécutions subies et de craintes de persécutions futures, une décision sans réelle motivation peut avoir des conséquences d'une extrême gravité. Lors de ces trois années, j’ai emmené cinq personnes aux urgences psychiatriques suite au rejet de leur demande. Chacune d'entre elles avait fait état dans leur récit, de violences, tortures, viols et autres mauvais traitements tous corroborés par un suivi médical et psychologique lourd. À la suite de ces événements, elles ont toutes été internées plus d'une semaine dans un hôpital psychiatrique. Les traumatismes subis par les persécutions vécues au pays sont comme «réactivés » par le rejet de la demande qui est interprété par les demandeurs, à juste titre, comme «la France ne croit pas aux violences que j'ai subies ». Ces conséquences, sur le plan de la reconstruction psychologique post-traumatique, sont simplement désastreuses.

\section{L'oubli de l'acte de persécution dans la demande d'asile}

Selon la définition donnée par la Convention de Genève, le réfugié est une personne qui «craint avec raison d'être persécutée». Cette crainte de persécutions est à la base de toute demande de protection. Elle est au premier plan pour les personnes qui sollicitent une demande d'asile, car elle est la raison de leur exil forcé. L'accueil que nous faisons en CADA traite prioritairement de cette problématique humaine et très souvent psychologique. L'objectif est de commencer les soins pour l'ensemble des blessures subies au pays le plus rapidement possible, car la réalité de certains demandeurs d'asile peut être comparable à celle de blessés de guerre. Nous avons accueilli des personnes venant de Syrie avec des éclats de munitions dans le corps et qui nécessitaient d'être réopérées. Des hommes de Tchétchénie défigurés par les tortures, ou encore des femmes de la République démocratique du Congo qui nécessitaient des opérations gynécologiques suite à la violence des nombreux viols subis. Aux côtés des stigmates physiques, il faut ajouter les stigmates invisibles, les symptômes posttraumatiques. Un exil forcé est forcément perturbant psychologiquement, mais les troubles post-traumatiques sont une dimension que la demande d'asile ne prend presque pas en compte. Plusieurs facteurs sont pourtant essentiels dans l'appréhension d'une procédure juste et équitable. La première problématique, commune à chaque demandeur d'asile, repose dans l'injonction paradoxale de la demande d'asile. Une personne doit avoir été persécutée ou craindre de l'être pour prétendre demander l'asile. Les persécutions ou craintes de persécutions vont forcément entraîner chez la victime un choc post-traumatique qui peut être suivi par des troubles. Ces troubles peuvent être des cauchemars, des reviviscences diurnes et nocturnes, des symptômes paranoïaques ou encore des troubles de la mémoire. Cependant, le demandeur d'asile sera dans l'obligation de faire état de l'ensemble de ces éléments de sa vie aussi personnels qu'ils soient, s'il souhaite obtenir le statut de réfugié. Car comme dit précédemment, la charge de la preuve pèse sur le demandeur qui est dans l'obligation d'exposer un récit de sa vie. On imagine bien les difficultés auxquelles sont confrontées ces personnes. Elles doivent, dans un récit, puis dans la durée d'un entretien, faire état des moments douloureux ou violents de leur vie comme une exécution, un bombardement, un viol ou une torture. De par les critères de reconnaissance imposés par l'OFPRA et la CNDA, les récits de vie doivent en plus être cohérents, compréhensibles, précis et détaillés. Nombreux sont les demandeurs d'asile suivis par un psychologue et un psychiatre que j'ai vus déboutés de leur demande d'asile car incapables de satisfaire l'ensemble de ces critères. En raison de leur situation de santé, et de leur prise en charge médicale, la majorité de ces déboutés ont obtenu un droit au séjour pour soins. Ce camouflet illustre l'incapacité des autorités d'élaborer une réelle expertise médicale, condition essentielle pour une procédure d'asile juste et équitable. 


\section{L'application du droit pénal en compétence universelle pour certains cas graves}

Cette insuffisance manifeste de la méthode d'instruction face aux conséquences des persécutions révèle clairement que l'objectif général n'est pas l'analyse de ces persécutions. Pourtant, certains dossiers de demande d'asile relèvent de crimes d'une telle gravité qu'il serait opérant de les analyser seulement au regard de ces actes. Une jeune Syrienne, présente lors du bombardement de son université à Alep, est bien une victime d'un crime de guerre. Ces crimes de guerre, de tortures, ou autres crimes graves, sont soumis à une compétence universelle en matière pénale. Certes en France, à l'inverse de la Belgique, nous avons restreint les possibilités pratiques de saisine mais le concept n'en est pas moins intéressant au regard de l'asile. En effet, sur une compétence pénale universelle pleine et non restreinte, un nombre de dossiers de demandeurs d'asile pourrait ouvrir le droit à une saisine. La Cour pénale internationale (CPI) repose aussi sur de nombreux témoignages de personnes qui sont réfugiées. Dans notre centre d'accueil, nous avons eu l'occasion d'accompagner un résident kényan sollicité par les autorités pour témoigner devant la CPI sur l'enquête des violences post-électorales au Kenya en 2007. Ces enquêtes extraordinaires sont certes restreintes en raison des moyens employés, mais elles donnent une dimension différente aux actes qui peuvent être soumis lors d'une demande d'asile. Que ce soit par une justice internationale ou sur la base d'une compétence universelle, les crimes graves subis par certains demandeurs d'asile sont déjà considérés par certains ordres juridiques comme des actes condamnables de manière extraterritoriale.

Les arguments de compétence territoriale de l'État souverain nous imposent de ne pas intervenir dans les crimes commis sur des étrangers, à l'étranger, par des étrangers. Cependant, l'ordre juridique international reconnaît un de ces principes essentiels qui est l'universalité de certains droits fondamentaux. Dans cette perspective, l'approche d'une demande d'asile serait une procédure de condamnation d'actes graves contraires à ces principes fondamentaux de notre ordre juridique international. Le souhait de nombreux demandeurs d'asile, que j'ai accompagnés, était de pouvoir saisir la Cour EDH. Cette démarche, que de nombreux demandeurs d'asile ont en commun, quelle que soit leur nationalité, est représentative d'une volonté d'obtenir une justice reconnaissant les crimes subis. La protection contre les actes futurs de persécution n'est pas recherchée par les demandeurs au travers de la procédure d'asile car la protection est pour eux déjà effective depuis qu'ils ont quitté le territoire où se trouvaient les menaces. Tandis que la reconnaissance des actes de persécution passés au pays est un enjeu primordial pour les demandeurs d'asile, la seule question effective dans la demande d'asile en France est la crainte future de persécutions en cas de retour. Lors de mes préparations d'audience, je devais sans cesse expliquer que l'objectif n'était pas de reconnaître la culpabilité de l'état d'origine mais d'établir les risques en cas de retour dans ce même pays. Ces demandeurs de justice ne peuvent qu'être déçus dans leur quête vitale lorsqu'ils aboutissent avec cette procédure d'asile qui les traite avant tout comme des immigrés. Car il suffit d'étudier les statistiques des pays d'origine pour voir le lien avec les pays qui vivent actuellement des crises profondes. Lorsque l'Ukraine a commencé sa guerre fratricide, nous avons accueilli moins de six mois après le premier afflux de demandeurs d'asile ukrainiens dans notre centre. Nous avons vu cette guerre qui a eu lieu aux frontières de l'Europe et celle qui ronge actuellement le Moyen-Orient. Dans l'ensemble des circonstances, les États européens, la France en tête, ont condamné les violations des droits fondamentaux au point de brandir une menace militaire. L'épisode de l'attaque aux armes chimiques en Syrie, et la volonté de la communauté internationale d'intervenir militairement pour y mettre fin, résume le traitement médiatique que peuvent opérer des choix politiques. En revanche, la seule réalité concrète généralement observée réside dans le sentiment général de compassion et de volonté de justice de l'opinion publique. Dans cette optique, on ne peut pas dire que la défense de droits universels pourrait souffrir d'un manque de soutien de la part de l'opinion publique. Il serait possible d'imaginer la mise en place pour certaines demandes d'une procédure pénale basée sur la compétence universelle qui serait exclusivement intéressée par la condamnation de crimes graves et la reconnaissance d'un statut protecteur de victime. Dès lors, la base d'application du droit d'asile ne peut plus être la personne. Nous avons démontré que ce vecteur de qualification sous-entend une approche du demandeur d'asile en tant qu'étranger et entraînait des vicissitudes structurelles dans le droit et la procédure d'asile. $\mathrm{Si}$, au contraire, nous utilisons comme vecteur de qualification juridique l'acte et non plus la personne, la procédure est ainsi concentrée sur les craintes de persécutions et les persécutions subies et non le statut administratif de la personne. Par conséquent, la procédure pénale en compétence universelle permet naturellement une meilleure prise en compte du traumatisme ou des blessures physiques. Les problématiques de politique d'immigration des autorités seraient effacées au profit des problématiques humanistes et universelles qui sont à la base de toute demande d'asile.

\section{VII.Une procédure d'asile sous forme de procédure pénale ad hoc?}

Si nous analysions la demande d'asile du point de vue de l'acte et non de la personne, nous traiterions d'un crime grave qui a été subi par une victime sollicitant une protection. Le changement sémantique doit résumer une approche méthodologique et philosophique. Ce qui doit être intéressant dans la demande d'asile ce n'est pas la personne étrangère mais le crime grave qu'elle a subi et celui qu'elle risque de subir. Portée par les valeurs à la base de la compétence universelle, cette approche pousserait 
l'analyse des actes de persécution et en prendrait ainsi la pleine dimension. En dehors des cas permettant l'application d'une compétence universelle en matière pénale, nous pourrions généraliser cette approche et donc cette méthode de traitement pour toutes les demandes d'asile. Une procédure, qui doit prendre exclusivement en compte les actes et qui peut analyser leur gravité, leur implication et leurs conséquences, pourrait s'apparenter à une procédure pénale. La procédure pénale s'applique lorsqu'une infraction a été commise et a donc pour vecteur de qualification l'acte et non une personne, un crime ou un délit énuméré dans le Code pénal. Cette procédure pénale ad hoc pourrait analyser les actes incriminés pour condamner les coupables et reconnaître une protection aux victimes. Les pouvoirs d'enquête seraient larges, étendus et non limités dans le temps. Ils prendraient en compte tous les moyens permettant de prouver un acte incriminé et reprendraient ainsi le principe pénal de la preuve par tous moyens. Ils pourraient examiner des témoignages, procéder à des expertises pour vérifier certaines preuves apportées par les parties, ou encore solliciter une expertise médicale pour établir les blessures tant physiques que psychologiques.

Les moyens pour obtenir une procédure juste et équitable capable d'analyser des actes graves, nous les connaissons car nous les avons établis en procédure pénale, pour tous les crimes commis sur le territoire ou hors du territoire pour les Français. Avec un vecteur de qualification de la demande d'asile basé sur l'acte et non la personne, nous serions face à des demandes concernant des crimes commis hors du territoire. Ces situations ainsi présentées amèneraient naturellement à reproduire une procédure d'asile qui pourrait ressembler à cette procédure pénale $a d$ hoc. Les autorités en charge de l'asile seraient en face de deux parties, un demandeur d'asile et l'état d'accueil. Ces parties pourraient débattre de manière contradictoire des éléments présentés par le demandeur. Il serait naturel de respecter l'ensemble des principes fondamentaux d'une instruction juste et équitable. Au besoin, une expertise médicale pourrait être demandée afin d'établir et prendre en compte judiciairement l'existence de blessures physiques ou psychologiques. Cela mettrait fin à la non prise en compte actuelle des certificats médicaux produits devant les autorités françaises et qui a fait l'objet de condamnations claires de la part de la Cour européenne des droits de l'homme ${ }^{18}$. L'instruction croiserait des informations déjà recueillies avec les éléments d'autres demandes d'asile pour vérifier la véracité des faits allégués. Elle pourrait faire témoigner toute personne ayant un lien avec les faits soumis par la demande d'asile. Elle ne serait plus ainsi dépendante mais surtout inquisitrice du demandeur d'asile.

Le respect des principes de la procédure pénale en matière d'asile permettrait de mettre en place les moyens suffisants pour une prise en charge juste et équitable des demandes d'asile. Avec cette approche basée sur l'acte et non la personne, la question du financement des moyens donnerait sûrement lieu à un débat politique, mais la question humaine et philosophique ne pourrait plus être détournée. Comment justifier, au regard de la gravité de certains crimes, qu'une victime non française n'aurait pas le même traitement judiciaire qu'une victime française ou une victime sur le territoire français, simplement parce que les crimes ont été commis hors de France. La gravité des crimes ne peut pas diminuer en raison de leur extraterritorialité. La demande d'asile devrait ainsi être une procédure pénale ad hoc à compétence universelle qui permettrait non seulement la reconnaissance du statut protecteur de réfugié, mais surtout la reconnaissance du statut de victime de crimes internationaux. De par mon expérience, les demandeurs d'asile ne nous posent pas la question de savoir si nous sommes en capacité de tous les accueillir. La véritable question est notre capacité de conscience universelle envers les autres, en raison de la violation de principes internationaux que nous considérons comme fondamentaux. Nous passerions ainsi de l'instruction de demandes d'asile déposées par des immigrés à l'établissement de faits allégués par des victimes potentielles de crimes graves. 